MODELING, IDENTIFICATION AND CONTROL, 1994, vOL. 15, NO. 4, 245-252

doi:10.4173/mic.1994.4.3

\title{
Optimal and adaptive control of underwater vehicles
}

\author{
SVEIN I. SAGATUN†
}

Keywords: Optimal control, adaptive control, underwater vehicle dynamics.

This article contains a continuous-time optimal and adaptive control scheme for underwater vehicles moving in six degrees of freedom. The control scheme is an extension of the algorithm of Johansson (1990) and a modification of the algorithm found in Sagatun (1992). The algorithm is optimal in the sense that it minimizes the state errors and the forces which contribute to the vehicle's kinetic energy that is spent to correct these errors. The performance measure does also contain a term which penalizes the quadratic tracking errors proportional to the rate of energy which dissipates from the system due to damping.

\section{Introduction}

Optimization is in the dictionary defined as: 'To make as perfect, effective or functional as possible'. Formally speaking, the optimal-control problem is to find the optimal control $u^{*}$ which minimizes a given performance functional $J(u)$. This functional is usually written as

$$
J(\tilde{z}, u)=S\left(x\left(t_{f}\right)\right)+\int_{t_{0}}^{t_{f}} L(\tilde{z}(\tau), u(\tau)) \mathrm{d} \tau
$$

where $\tilde{z}$ is the system state we wish to control. It is well known from the literature, e.g. Athans and Falb (1966), that the optimal control $u_{x}^{*}(t)$ for the state space we want to control with the performance measure in (1) is found by solving the following equation for all $t$

$$
\frac{\partial J^{*}(\boldsymbol{z}, t)}{\partial t} \min _{\boldsymbol{u}_{\mathbf{x}}(t)}\left\{L\left(\tilde{\mathbf{z}}(t), \boldsymbol{u}_{\mathbf{x}}(t)\right)+\frac{\partial J^{*}(\tilde{\boldsymbol{z}}, t)^{\mathrm{T}}}{\partial \tilde{\boldsymbol{z}}} \dot{\tilde{\mathbf{z}}}\right\}=0
$$

Hence, the problem is to find the function $J^{*}(\tilde{z}, t)$ which is called the Hamilton principle function of optimization.

\section{Kinematics}

This article uses an earth-fixed coordinate system sometimes denoted the inertial frame and a vehicle fixed system. We will use the SNAME convention, SNAME (1950) for the placement of the coordinate systems. Position and orientation will be represented by Euler angles and inertial-fixed coordinates $\boldsymbol{x}=[x, y, z, \phi, \theta, \psi]^{\mathrm{T}} \in \mathscr{R}^{6}$ while we will only deal with vehicle-fixed velocities and accelerations denoted $\dot{\boldsymbol{v}}=[u, v, w, p, q, r]^{\mathrm{T}} \in \mathscr{R}^{6}$ and $\dot{\boldsymbol{v}} \in \mathscr{R}^{6}$.

Received 28 January 1994.

† ABB Industry AS Department of Marine Automation, N0501 Oslo, Norway.

This paper was presented at the 1993 American Control Conference, June 2-4, 1993, San Francisco, CA., and is reprinted with kind permission from the American Automatic Control Council. 
The earth-fixed velocities and rate of change of Euler angles $\dot{\boldsymbol{x}}=[\dot{x}, \dot{y}, \dot{z}, \dot{\phi}, \dot{\theta}, \dot{\psi}]^{\mathrm{T}}$ can be transformed to a vehicle-fixed velocity vector $\boldsymbol{v}$ by using the transformation matrix $\boldsymbol{J}(\boldsymbol{x})$, the so called Jacobian matrix. Hence, the transformation from the vehicle-fixed frame to the inertial frame can be performed by employing the following relations

$$
\begin{aligned}
& v=J^{-1}(\boldsymbol{z}) \dot{\boldsymbol{X}} \\
& \dot{v}=\boldsymbol{J}^{-1}(\boldsymbol{x})\left(\ddot{\boldsymbol{x}}-\dot{J}(\mathbf{x}) \boldsymbol{J}^{-1}(\boldsymbol{x}) \dot{\boldsymbol{x}}\right)
\end{aligned}
$$

for a nonsingular $\boldsymbol{J}(\boldsymbol{x})$ matrix. Notice that the vector $\boldsymbol{v}$ is not generalized coordinates, while the vector $\boldsymbol{x}$ represents proper generalized coordinates.

While it is most common to express the equations of motion in the vehicle-fixed frame, the formulation in the inertial frame has some advantages. One is that the differentiation of position, with respect to time, yields velocity directly without going through the Jacobian matrix $\boldsymbol{J}(\boldsymbol{x})$. This property is necessary if we want to employ the Euler-Lagrange equation and is of great advantage in proofs of stability and optimality.

\section{The vehicle model}

The kinetic energy for the vehicle and its ambient water is in the inertial-fixed frame formulation gives by

$$
T_{x}=\frac{1}{2} \dot{\boldsymbol{X}}^{T} \boldsymbol{M}(\boldsymbol{x}) \dot{\boldsymbol{X}}
$$

where $\boldsymbol{M}(\boldsymbol{x})=\boldsymbol{J}^{-\mathbf{T}}(\boldsymbol{x}) \boldsymbol{M}_{q} \boldsymbol{J}^{-1}(\boldsymbol{x})$ and $\boldsymbol{x}$ is a generalized coordinate vector. $\boldsymbol{M}_{q}$ is the mass of the vessel and its added inertia formulated in the vehicle-fixed frame. We can formulate the Euler-Lagrange equations for the 'rigid body-ambient water' system in the vehicle-fixed frame:

$$
\tau_{x_{i}}=\frac{\mathrm{d}}{\mathrm{d} t}\left(\frac{\partial T_{x}}{\partial \dot{x}_{i}}\right)-\frac{\partial T_{x}}{\partial x_{i}}+\frac{\partial \mathscr{F}_{d}}{\partial \dot{x}_{i}} ; \quad i=1 \ldots n
$$

which yields

$$
\tau=M(x) \ddot{x}+\dot{M}(x, \dot{x}) \dot{x}-\frac{1}{2} \frac{\partial}{\partial x}\left(x^{\mathrm{T}} M(x) \dot{x}^{\mathrm{T}} \times D(x, \dot{x}) \dot{x}\right.
$$

or

$$
\tau=M(x) \ddot{x}+C(x, \dot{x}) \dot{x}+D(x, \dot{x}) \dot{x}
$$

where $\boldsymbol{D}$ is the vehicle's damping matrix formulated in the vehicle-fixed frame. Recall that $\dot{M}$ equals to 0 . It is useful to define the matrix $\boldsymbol{C}(z, \dot{\boldsymbol{x}})$ as

$$
C(x, \dot{x})=\frac{1}{2} \dot{M}(x)+N(x, \dot{x})
$$

where $N(x, \dot{x})$ is defined such that

$$
N(x, \dot{x}) \dot{x}=\frac{1}{2} \dot{M}(x) \dot{x}-\frac{1}{2} \partial x\left(\dot{x}^{\mathrm{T}} M(x) \dot{x}\right.
$$

It is easy to prove that $N(x, \dot{x})$ is such that $\dot{\boldsymbol{x}}^{\mathrm{T}} \mathbf{N}(\mathbf{x}, \dot{\boldsymbol{x}}) \dot{\boldsymbol{x}}=0 \forall \dot{\boldsymbol{x}}(t)$. The physical interpretation of this is that the $N_{x}(x, \dot{x})$ matrix represents the workless forces of the vehicle Sagatun (1992) and Sagatun and Fossen (1981). 
We will, in the rest of this article, assume that potential energy, i.e. the effect of gravity, is included in the $\tau$ vector such that $\tau=\tau_{o x}-\boldsymbol{g}(\boldsymbol{x})$ is the vector representing forces and moments acting on the vehicle due to gravity.

\section{Optimal criteria}

Sagatun (1992) presents an adaptive and optimal controller for a mechanical manipulator which minimizes the tracking errors and the forces which are needed to correct these errors. Only the forces which contribute to the manipulator's kinetic energy are minimized. There is no point in optimizing the forces that contribute to the potential energy since the potential energy is end-point dependent only. This article presents several modifications of the control scheme introduced in Johansson (1990). We employ a performance measure which, in addition to the kinetic energy also includes the energy which dissipates away from the vehicle due to damping effects from the water, e.g. viscous friction. The work done by the system when subtracting potential energy becomes

$$
W=\int_{t_{0}}^{t_{f}} \dot{\boldsymbol{x}}^{\mathrm{T}}\left(\boldsymbol{M} \ddot{\mathbf{x}}+\frac{1}{2} \dot{\boldsymbol{M}} \dot{\boldsymbol{x}}+\boldsymbol{N} \dot{\boldsymbol{x}}+\boldsymbol{D} \dot{\mathbf{x}}\right) \mathrm{d} t
$$

The term $\dot{\boldsymbol{x}}^{\mathrm{T}} \boldsymbol{N}_{x} \dot{\boldsymbol{x}}$ is evaluated to zero. A natural extension of the control variable found in Johansson (1990) is therefore for underwater robots

$$
\boldsymbol{u}=M_{x} T_{1} \dot{\tilde{z}}+\left(D_{x}+\frac{1}{2} \dot{M}_{x}\right) T_{1} \tilde{z}
$$

since the dissipative effect (i.e. viscous damping) is very important for marine vehicles. The vector $\tilde{\boldsymbol{z}}$ is defined as $\tilde{\boldsymbol{z}}=\left[\left(\dot{\boldsymbol{z}}-\dot{\boldsymbol{z}}_{r}\right)^{\mathrm{T}},\left(\boldsymbol{x}-\boldsymbol{X}_{r}\right)^{\mathrm{T}}\right]^{\mathrm{T}}$ and the matrix $\boldsymbol{T}_{1}$ is the upper $n \times 2 n$ matrix of $\boldsymbol{T}_{0}$ defined as

$$
T_{0}=\left[\begin{array}{l}
T_{1} \\
T_{2}
\end{array}\right]=\left[\begin{array}{ll}
T_{11} & T_{12} \\
0_{n \times n} & I_{n \times n}
\end{array}\right]
$$

The introduction of the $\boldsymbol{T}_{0}$ matrix results in a non-physical interpretation of the $\boldsymbol{u}$ vector since the matrices are multiplied with a linear combination of acceleration and velocity, and velocity and position respectively. An advantage with the use of the $\boldsymbol{T}_{\mathbf{0}}$ matrix is that the new control variable also becomes a function of the position errors.

\section{Basic assumptions}

The following assumptions are made in the derivation of the control schemes in this article. Assumption A8 is relaxed when the adaptive controller is derived.

A1 The motion is in the inertial reference frame governed by the equation (5).

A2 The reference trajectory is smooth, bounded and within kinematic and physical limits. This can be achieved by assuming that the desired reference trajectory is generated by the strictly stable reference model

$$
\ddot{\boldsymbol{x}}_{r}+K_{d} \dot{\boldsymbol{x}}_{r}+K_{p} \mathbf{x}_{r}=K_{p} \mathbf{x}_{d}
$$

The $n \times n$ matrices $\boldsymbol{K}_{d}$ and $\boldsymbol{K}_{p}$ are defined such that $\ddot{\boldsymbol{x}}_{r}, \dot{\boldsymbol{x}}_{r}$ and $\boldsymbol{x}_{r}$ are within the physical limits of the vehicle. Physical limited accelerations and velocities imply that $\ddot{\boldsymbol{x}}_{r}, \dot{\boldsymbol{x}}_{r}$ and $\boldsymbol{x}_{r}$, are $\in L^{\infty}$ and $\boldsymbol{x}_{r} \in C^{1}$.

A3 The state variables we use in the presented control algorithm are defined by the vector $\boldsymbol{z}=\left[\dot{\boldsymbol{x}}^{\mathrm{T}}, \boldsymbol{x}^{\mathrm{T}}\right]^{\mathrm{T}}$ and the tracking error vector $\tilde{\boldsymbol{z}}=\left[\left(\boldsymbol{z}-\dot{\boldsymbol{x}}_{\mathrm{r}}\right)^{\mathrm{T}}\right.$, $\left.\left(\boldsymbol{x}-\boldsymbol{X}_{r}\right)^{\mathrm{T}}\right]^{\mathrm{T}}, \tilde{\boldsymbol{z}} \in \mathscr{R}^{2 n}$. It will also be useful to define the vector $\boldsymbol{Z}_{r}=\left[\dot{\boldsymbol{x}}_{r}^{\mathrm{T}}, \boldsymbol{x}_{r}^{\mathrm{T}}\right]^{\mathrm{T}}$. 
A4 The control variable to be minimized is given by (8).

A5 All states are measured, that is vehicle fixed velocities $v$ and inertial positions and orientations $\boldsymbol{x}$

A6 In the discussion of the controller, the $y$ vector is referred to as the measurement vector, see also A5. A useful transformation in the coming discussion is $\boldsymbol{z}=\boldsymbol{T} \boldsymbol{y}$ where the transformation matrix $\boldsymbol{T}$ is given as $T=\operatorname{diag}\left(\boldsymbol{J}(\boldsymbol{x}), \boldsymbol{I}_{n \times n}\right)$.

A7 The structure of the equations of motion are completely known and linear in its parameters.

A8 The parameters in the equations of motion are completely known.

A9 We will use the term global stability when we use Jacobian matrices, even though, it is not mathematically correct from a rigorous point of view, since the Jacobian matrices may become singular.

\section{The optimal control algorithm}

This section presents extensions of the algorithm presented in Johansson (1990). Global uniformly asymptotic stability is proven for the case of a perfectly known vehicle model. However, this is rarely the case for underwater vehicles, so an adaptive version of the algorithm is also presented. Global asymptotic stability is proven for the tracking errors and a bounded parameter estimate is guaranteed in the adaptive case.

\subsection{State-space representation}

A state-space description of (5) combined with the control variable in (8) expressed in the $\tilde{\boldsymbol{z}}$ space can be found according to

$$
\dot{\tilde{\mathbf{z}}}=\boldsymbol{T}_{0}^{-1}\left[\begin{array}{cc}
-M^{-1}(\boldsymbol{C}+\boldsymbol{D}) & \mathbf{0}_{n \times n}-\boldsymbol{T}_{11}^{-1} \\
\boldsymbol{T}_{11}^{-1} & \boldsymbol{T}_{12}
\end{array}\right] \boldsymbol{T}_{0} \tilde{\boldsymbol{z}}+\boldsymbol{T}_{0}^{-1}\left[\begin{array}{c}
\boldsymbol{M}^{-1} \\
\mathbf{0}_{n \times n}
\end{array}\right] \boldsymbol{u}
$$

\subsection{The control objective}

The control objective is to minimize the quadratic performance index given by (1) with the Lagrangian

$$
L(\tilde{\boldsymbol{z}}, \boldsymbol{u})=\frac{1}{2} \tilde{\boldsymbol{z}}^{\mathrm{T}}\left(\boldsymbol{Q} \boldsymbol{T}_{0}^{\mathrm{T}} \boldsymbol{E} \boldsymbol{D}(\boldsymbol{z}) \boldsymbol{E}^{\mathrm{T}} \boldsymbol{T}_{0}\right) \tilde{\mathbf{z}}+\frac{1}{2} \boldsymbol{u}^{\mathrm{T}} \boldsymbol{R} \boldsymbol{u}
$$

where $\boldsymbol{R}=r_{1} \cdot \boldsymbol{I}_{m \times m}=\boldsymbol{R}^{\mathrm{T}}>0, \boldsymbol{E}=\left[\boldsymbol{I}_{n \times m}, \mathbf{0}_{n \times n}\right]^{\mathrm{T}}, \boldsymbol{Q}=\boldsymbol{Q}^{\mathrm{T}}>0$, and $t_{f}$ is not fixed. A diagonal structure of the matrix $\boldsymbol{R}$ given above is assumed since energy spent to correct tracking errors in one direction is as valuable as in another one. The weighting of the distribution of energy to each of the vehicle's thrusters is taken care of in (22). It is important to realize that we minimize the velocity and thrust in the inertial coordinate system.

\subsection{Optimality and stability}

It is well known from the literature, e.g. Athans and Falb (1966), that the optimal control $u_{x}^{*}(t)$ for the state equation in (10) with the performance measure in (1) can be found by solving the Hamilton-Jacobi equation given by (2). 
Lemma 1

The following function $\boldsymbol{J}^{*}$ satisfies the Hamilton-Jacobi equation and constitutes a Hamilton's principal function for the optimization problem formed by (2) and (11) under the assumptions made above

$$
J^{*}(\tilde{\boldsymbol{Z}})=\frac{1}{2} \tilde{\boldsymbol{Z}}^{\mathrm{T}}(t) \boldsymbol{T}_{0}^{\mathrm{T}}\left[\begin{array}{ll}
\boldsymbol{M}(\boldsymbol{x}) & \mathbf{0}_{n \times n} \\
\mathbf{0}_{n \times n} & \boldsymbol{K}
\end{array}\right] \boldsymbol{T}_{0} \tilde{\boldsymbol{Z}}(t)
$$

where $\boldsymbol{K}$ is a positive definite symmetric matrix $K \in \mathscr{R}^{n \times n}$ and for $\boldsymbol{K}$ and $\boldsymbol{T}_{0}$ solving the matrix equation

$$
\left(\begin{array}{ll}
\boldsymbol{0}_{n \times n} & \boldsymbol{K} \\
\boldsymbol{K} & \mathbf{0}_{n \times n}
\end{array}\right)+\boldsymbol{Q}-\boldsymbol{T}_{0}^{\mathrm{T}} \boldsymbol{E} \boldsymbol{R}^{-1} \boldsymbol{E}^{\mathrm{T}} \boldsymbol{T}_{0}=0
$$

The optimal feedback law $\boldsymbol{u}^{*}$ that minimizes (1) with the Lagrangian in (11) is

$$
u^{*}(t)=-\boldsymbol{R}^{-1} \boldsymbol{E}^{\mathrm{T}} \boldsymbol{T}_{0} \tilde{\mathbf{z}}(t)
$$

Proof: The proof is rather lengthy. A complete proof is found in Sagatun (1992).

Theorem 1

The system described by (10) and controlled by (14) always globally uniformly asymptotically stable with the choice of the weighting matrices $\boldsymbol{T}_{0}$ and $\boldsymbol{K}$ given in Johnsson (1990) and the above assumptions.

Proof: The theorem is proved if we can find a suitable Lyapunov function candidate $V(\tilde{z}, t)$ for the nonautonomous system described by (10) satisfying, Lee and Markus (1967):

(i) $V(\tilde{z}, t)$ is continuous at $\tilde{\boldsymbol{z}}=0 \forall t$,

(ii) $V(\tilde{z}, t)$ is positive radially growing with $\|\tilde{z}\|$,

(iii) $V(\tilde{\mathbf{z}}, t)$ has a unique minimum at the origin of the error space and

(iv) $V(\tilde{z}, t)$ is negative definite along $\tilde{\boldsymbol{z}}$ and $t$.

It is straightforward to show that $h \tilde{\mathbf{z}}, t)=J^{*}(\tilde{\mathbf{z}}, t)$ satisfies the three first requirements. The last requirement is also easily proven since (2) states that

$$
\begin{aligned}
& \frac{d V}{d t}=\frac{\partial J^{*}(\tilde{\mathbf{z}}, t)}{\partial t}+\frac{\partial J^{*}(\tilde{\mathbf{z}}, t)^{\mathrm{T}}}{\partial \tilde{\boldsymbol{z}}} \dot{\tilde{\mathbf{z}}}=-L(\tilde{\boldsymbol{Z}}(t), \boldsymbol{u}(t)) \\
& \quad \Downarrow \\
& \frac{d V}{d t} \leqslant 0 \quad \forall \tilde{\boldsymbol{Z}}(t) \neq 0
\end{aligned}
$$

Hence we have shown that the system described by (10) and controlled by (14) is always globally uniformly asymptotically stable. This concludes the proof.

Comment 1. The dissipative forces in (11), represented by the $D(z)$ matrix, increase the stability of the system. This can also be seen when we look on the mapping from the vehicle's thrust $\tau$ to velocity $\dot{\boldsymbol{x}}$ written as $\tau \rightarrow \dot{\boldsymbol{X}}$ which is dissipative since $\dot{\boldsymbol{V}}=\boldsymbol{\tau}^{\mathrm{T}} \dot{\boldsymbol{x}}-\dot{\boldsymbol{x}}^{\mathrm{T}}$ $\boldsymbol{D}(\boldsymbol{z}) \dot{\boldsymbol{x}}$, (Sagatun and Fossen 1991). We observe from the expression of $V$ that the gradient of $V$ becomes more negative, that is $V$ converges faster to zero, with increased damping. This, of course expected compared to the corresponding linear case 
where the phase margin increases with increased damping. The positive term $\tilde{\boldsymbol{z}}(t)^{\mathrm{T}} \boldsymbol{T}_{0}^{\mathrm{T}} \boldsymbol{E} \boldsymbol{D}(\boldsymbol{z}) \boldsymbol{E}^{\mathrm{T}} \boldsymbol{T}_{0} \tilde{\boldsymbol{z}}(t)$ with $\boldsymbol{T}_{12}=0, \boldsymbol{T}_{11}=\boldsymbol{I}_{n \times n}$ corresponds to the rate the energy dissipates from the vehicle.

\subsection{The control law}

A combination of the control variable in (8) with the state-space representation in (10) yields the following expression for the resulting thruster forces

$$
\tau^{*}=C \dot{\boldsymbol{x}}+D \dot{\boldsymbol{x}}+M\left[\ddot{x}_{r}-T_{11}^{-1} T_{12} \dot{\tilde{x}}-T_{11}^{-1} M^{-1}\left((C+D) E^{\mathrm{T}} T_{0} \dot{\boldsymbol{z}}-u^{*}(t)\right)\right]
$$

where $\boldsymbol{u}_{x}^{*}$ is given by (14). This expression is considerably simplified if we also assume that the $\boldsymbol{Q}$ matrix can be written on the form $\boldsymbol{Q}=\operatorname{diag}\left[q_{1} \boldsymbol{I}_{n \times n}, q_{2} \cdot \boldsymbol{I}_{n \times n}\right] . \boldsymbol{T}_{11}$ and $\boldsymbol{T}_{12}$ will then become diagonal matrices such that $\boldsymbol{T}_{11}=\boldsymbol{t}_{11} \cdot \boldsymbol{I}_{n \times n}$ and $\boldsymbol{T}_{12}=t_{12} \cdot \boldsymbol{I}_{n \times n}$. The new control law then becomes

$$
\tau^{*}=M\left(\ddot{\boldsymbol{X}}_{r}-\frac{t_{12}}{t_{11}} \dot{\hat{\boldsymbol{X}}}+(C+D)\left(\dot{\boldsymbol{X}}_{r}-\frac{t_{12}}{t_{11}} \tilde{\boldsymbol{x}}\right)+\frac{u^{*}}{t_{11}}\right.
$$

This expression can be simplified even more by defining the signals

$$
\begin{aligned}
& S(t)=t_{11} \dot{\boldsymbol{X}}_{r}-t_{12} \tilde{\boldsymbol{X}} \\
& \dot{\boldsymbol{S}}(t)=t_{11} \ddot{\boldsymbol{X}}_{r}-t_{12} \tilde{\boldsymbol{X}}
\end{aligned}
$$

and performing the parameterization

$$
\psi(\dot{\boldsymbol{s}}, \boldsymbol{s}, \dot{\boldsymbol{x}}, \mathbf{x}) \theta=\frac{1}{t_{11}}(M \dot{s}+C s+D s)
$$

and $u_{t_{1}}^{*}(t)=\frac{1}{t_{11}} u(t)$ such that

$$
\tau^{*}=\psi(\dot{s}, \boldsymbol{s}, \dot{\boldsymbol{x}}, \boldsymbol{x}) \boldsymbol{\theta}+\boldsymbol{u}_{t_{11}}^{*}(t)
$$

Notice the similarity between the error signal $s(t)$ and the one employed by Fossen and Sagatun (1991). The control law in (17) is of no practical use, since the resulting thrust forces are calculated in an inertial reference frame. Eqn. (17) is also impractical in the sense that they use velocities decomposed in an inertial reference frame while we measure the vehicle-fixed velocities. A last drawback with (17) is that it is much more complicated in the inertial-frame than in the vehicle-fixed frame. It is, however, possible to transform equation (17) to the vehicle-fixed frame by using the following lemma.

\section{Lemma 2}

The equation described by (17) can be transformed to a vehicle-fixed coordinate system by employing the following transformation:

$$
J^{\mathrm{T}}(x) \psi(\dot{s}, s, \dot{\boldsymbol{x}}, \boldsymbol{x}) \boldsymbol{\theta}=\psi_{q}\left(\dot{s}_{q}\left(\dot{s}_{q}, s_{q}, v\right) \theta_{q}\right.
$$

where the regressor matrix $\psi_{q}$ is found from

$$
\psi_{q}\left(\dot{s}_{q}, s_{q}, v\right) \theta_{q}=\frac{1}{t_{11}}\left(M_{q} \dot{s}_{q}+C_{q}(v) s_{q}+D_{q}(v) s_{q}\right)
$$


where $\dot{\boldsymbol{s}}_{q}$ and $\mathbf{s}_{q}$ is defined in (21). The resulting control law formulated in the vehicle fixed coordinate system then becomes

$$
\tau_{q}^{*}=\psi_{q}\left(\dot{s}_{q}, s_{q}, v\right) \theta_{q}+u_{q}^{*}
$$

The feedback control law formulated in the vehicle-fixed frame $u_{q}^{*}(t)$ is found from

$$
u_{q}^{*}(t)=-\frac{1}{t_{11}} J^{\mathrm{T}}(x) R^{-1} E^{\mathrm{T}} \boldsymbol{T}_{0} T \tilde{y}
$$

Proof: The new signals $\dot{\boldsymbol{s}}_{q}$ and $s_{q}$ are found by using (3) on each term in (16) such that

$$
\begin{aligned}
& s_{q}(t)=t_{11} v_{r}-t_{12} J^{-1}(x) \tilde{x} \\
& \dot{S}_{q}(t)=t_{11} \tilde{v}_{r}-t_{12}\left(\dot{J}^{-1}(x) \tilde{x}+v\right)
\end{aligned}
$$

The rest of the proof is straightforward.

Note that the first term in $\dot{\boldsymbol{s}}_{q}$ and $\boldsymbol{s}_{q}$ are feed-forward terms while the latter are feedback terms. The reparameterization, by using a virtual vector in (18), is similar to the one employed in Fossen and Sagatun (1991). Notice also that all signals in (21) are measurable or preprogrammed. The form of the $\psi_{q}$ vector is also much simpler than the $\psi$ vector.

The corresponding commanded angular velocities $\boldsymbol{n}_{\mathrm{d}}$ to each thruster can be computed from

$$
\boldsymbol{n}_{d}=\boldsymbol{B}_{t}^{\dagger}(\boldsymbol{v}) \tau_{q}^{*}
$$

where $\boldsymbol{B}_{t}^{\dagger}(\boldsymbol{v})=\boldsymbol{W}^{-1} \boldsymbol{B}_{t}^{\mathrm{T}}\left(\boldsymbol{B}_{t}^{\mathrm{T}} \boldsymbol{W}^{-1} \boldsymbol{B}_{t}\right)^{-1}$ for $m>n$ and $\boldsymbol{B}_{t}^{\dagger}=\boldsymbol{B}_{t}^{-1}(\boldsymbol{v})$ when $\boldsymbol{B}_{t}^{\dagger}(\boldsymbol{v})$ is nonsingular. We have used a positive and diagonal $\boldsymbol{W}$ which distributes energy between the different thrusters after a quadratic cost criterion.

\section{The adaptive control algorithm}

Many of the parameters in the $\boldsymbol{M}, \boldsymbol{D}(\boldsymbol{v})$ and $\boldsymbol{C}(\boldsymbol{v})$ matrices are unknown and timevarying for marine vehicles and especially for small underwater vehicles. This motivates the use of an adaptive version of (19).

Rewrite (19) such that it is expressed as a function of the current estimate of the parameters

$$
\tau_{q}=\psi_{q}\left(\dot{s}_{q}, s_{q}, v\right) \hat{\theta}_{q}+\psi_{q_{0}}\left(\dot{s}_{q}, s_{q}, v\right)+u_{q}^{*}(t)
$$

where $\left({ }^{*}\right)$ denotes the estimated value of $(\cdot)$ and $\psi_{0}$ represents the part of the model which is completely known. The effective control variable $\boldsymbol{u}_{q}$ is found by comparing (23) and (19) to be

$$
u_{q}=u_{q}^{*}+\psi_{q}\left(\dot{s}_{q}, \dot{s}_{q}, s_{q}, v\right) \partial_{q}
$$

where $\psi_{q} \in \mathscr{R}^{n \times p}$ and $\widetilde{\theta}_{q} \in \mathscr{R}^{p}, p$ is the number of parameters we want to adapt. The corresponding new feedback law formulated in the inertial frame becomes

$$
u_{t_{11}}(t)=u_{t_{11}}^{*}(t)+\psi(\dot{s}, s, \dot{x}, x) \tilde{\theta}
$$

We define the augmented error vector $e \in \mathscr{R}^{2 n+p}$ such that $\boldsymbol{e}=\left[\dot{\boldsymbol{Z}}^{\mathrm{T}}, \boldsymbol{\theta}^{\mathrm{T}}\right]^{\mathrm{T}}$ and introduce the new Lyapunov function candidate, Johansson (1990):

$$
\left.V_{e}(\boldsymbol{e}, t)=И \tilde{\boldsymbol{z}}, t\right)+V_{\theta_{q}}\left(\tilde{\boldsymbol{\theta}}_{q}\right)
$$

where $\boldsymbol{V}_{\theta_{q}}\left(\tilde{\boldsymbol{\theta}}_{q}\right)=\widetilde{\boldsymbol{\theta}}_{q}^{\mathrm{T}} \boldsymbol{K}_{\theta} \widetilde{\boldsymbol{\theta}}_{q}$ and $\boldsymbol{K}_{\theta}=\boldsymbol{K}_{\theta}^{\mathrm{T}}>0$ and $V(\tilde{\mathbf{z}}, t)$ is found in Theorem 1. We are now ready to formulate the following theorem: 
Theorem 2

The system described by (23) with unknown parameters and controlled by (24) is always globally asymptotically stable with respect to $\tilde{\boldsymbol{y}}$ with the following adaptation law

$$
\dot{\hat{\theta}}_{q}=-K_{\theta}^{-1} \psi_{q}^{\mathrm{T}} J^{-1}(\boldsymbol{x}) \boldsymbol{E}^{\mathrm{T}} \boldsymbol{T}_{\mathbf{0}} \boldsymbol{T} \tilde{y}
$$

Furthermore, the adaptive control law in (27) is stable in the sense that all parameters remain bounded for all $t$. The adaptive controller will be an optimal adaptive control system for constant parameters when $\widetilde{\theta}_{q}=0$ and under the assumptions made in the previous section.

Proof: If we differentiate (26) with respect to time we get

$$
\dot{\boldsymbol{V}}_{e}(\boldsymbol{e}, t)=\dot{\boldsymbol{V}}(\tilde{\mathbf{Z}}, t)+\dot{\boldsymbol{V}}_{\theta_{q}}\left(\tilde{\boldsymbol{\theta}}_{q}\right)
$$

A combination of (28) with the control law in (25) and the results from Johansson (1990) and Sagatun (1992) yield

$$
\dot{V}_{e}(e, t) \leqslant 0
$$

Notice that we assume constant parameters, i.e. $\dot{\theta}=0$. We use the adaptation law in (27) to eliminate terms containing $\boldsymbol{\theta}$ and $\dot{\theta}$. Hence we have proved that $V(e, t) \leqslant 0$. This implies that $V(t) \leqslant V(0)$ which, in turn, implies that $\tilde{\boldsymbol{z}}=\boldsymbol{T} \tilde{\boldsymbol{y}}$ is bounded. Bounded reference trajectories and the fact that $\psi_{q}, \boldsymbol{\theta}$ and $\boldsymbol{J}(\boldsymbol{x})$ are continuous bounded functions ensure that $V(e, t)$ as bounded, consequently $\dot{V}(e, t)$ is uniformly continuous in time. Finally, application of Barbălat's lemma (Barbălat 1959) shows that $\dot{V}(e, t) \rightarrow 0$ implying that $\tilde{\boldsymbol{z}}=\boldsymbol{T} \tilde{y}$ converges to zero and that $\hat{\boldsymbol{\theta}}_{q}$ remains bounded for all $t$. We have $\boldsymbol{u}_{q}=\boldsymbol{u}_{q}^{*}$ in the case of no parameter errors and, hence, we have an optimal controller with respect to the performance criterion in (1).

\section{ACKNOWLEDGMENTS}

The author wishes to express his gratitude to Dr. Rolf Johansson and Dr. Thor $\mathbf{I}$. Fossen for helpful discussions. Parts of this research has been funded by an Underwater Technology Grant from BP Exploration Norway AS.

\section{REFERENCES}

Athans, M. and Falb, P. L., eds (1966). Optimal Control (McGraw-Hill Book Company, New York).

BARBĂLAT (1959). Systèmes d'èquations diffèrentielles d'oscillations non linèaires. Revue de Mathématiques Pures et Appliquèes, 4, 267-270.

Fossen, T. I. and SAgatun, S. I. (1991). Adaptive Control of Nonlinear Underwater Robotic Systems. In Proceedings of the IEEE Conference on Robotics and Automation, pp. 1687-1695, Sacramento, California, April 1991.

JoHANSSON, R. (1990). Quadratic Optimization of Motion Coordination and Control. IEEE Trans. Automatic Control, 35, 1197-1208.

LeE, E. B. and Markus, L. (1967). Foundation of Optimal Control Theory (Wiley, New York).

SAGATUN, S. I. (1992). Modelling and Control of Underwater Vehicles: a Lagrangian approach, Dr. thesis. ITK report 1992:28-W NTH, Trondheim, Norway. June 1992

Sagatun, S. I. and Fossen, T. I. (1991). Lagrangian Formulation of Underwater Vehicles' Dynamics. In Proceedings of the IEEE IC SMC, pages 1029-1034, Charlottesville, VA, October 13-16, 1991.

SNAME. (1950). The Society of Naval Architects and Marine Engineers. Nomenclature for Treating the Motion of a Submerged Body Through a Fluid. In Technical and Research Bulletin 1-5. 